\title{
La "visión del aura" como experiencia alucinatoria en individuos no-clinicos
}

\author{
Alejandro Parra' - Universidad Abierta Interamericana
}

\begin{abstract}
Resumen
Individuos que dicen ver el aura/energía en forma espontánea también tienden a tener un nivel más elevado de actividad imaginativa/fantaseosa o de propensidad a la fantasía. Hay indicadores que muestran que quienes experimentan alta capacidad de absorción, usualmente experimentan también diversas formas parasomáticas, alucinaciones táctiles, y sensaciones de energías sutiles, asi como también ideación esquizotípica, error perceptual, y mayor sinestesia. El objetivo es correlacionar puntajes de absorción psicológica, disociación, propensidad a la fantasía, intensidad de la imaginería, y tendencia a la esquizotipia en individuos que dicen ver el aura $(\mathrm{N}=83)$ en comparación con un grupo sin experiencias $(\mathrm{N}=503)$. La muestra incluyó 678 estudiantes de psicología de ambos sexos, $449(76,5 \%)$ mujeres y $139(23,5 \%)$ varones, cuya rango etario es de 17 y 57 años $($ Media $=25,54 ; S D=7,22)$ residentes en la Ciudad de Buenos Aires y alrededores. Ningun estudiante recibió compensación económica. Respecto a la experiencia de ver el aura, se formuló la pregunta de la siguiente manera: "¿Ha tenido alguna experiencia usted es capaz de ver campos de energia o luces alrededor del cuerpo de una persona? (si duda, por favor, responda no)". Si la respuesta es afirmativa, la pregunta también mapea tres dimensiones de la experiencia: frecuencia de la experiencia, explicación subjetiva (racional, desconocida y paranormal) de la experiencia, y positivo o negativo impacto (emocional) en una escala Likert, siendo 1 positivo y 7 negativo. Los resultados muestran mayor nivel de esquizotipia cognitivo perceptual en comparación con quienes no tienen la experiencia. Posiblemente, estas personas tienen mucha más intensa vida imaginativa. Esta experiencia no necesariamente posee un significado psicopatológico para el individuo; quienes ven el aura simplemente podrían ser sensibles a las experiencias perceptuales anómalas.

Palabras Clave: Aura; Propensidad a la fantasía; Absorción; Formas parasomáticas; Imaginería.
\end{abstract}

\section{The aura experience as cognitive perceptual schizotypy in non-clinical population}

\begin{abstract}
Individuals who claim being able to spontaneously see the "aura" or energy field of a person, also tend to exhibit elevated levels of imaginative processing or fantasy proneness. There are some indications that those who have a high capacity for absorption usually also experience different parasomatic shapes, tactile hallucinations and sensations of subtle energies, as well as schizotypical ideation, perceptual error and increased synesthesia. The objective is to calculate the correlations of psychological absorption scores, dissociation, fantasy proneness, intensity of imagination and the schizotypical tendency of people who claim to see auras $(\mathrm{N}=83)$, in comparison to a group of people lacking these experiences $(\mathrm{N}=503)$. The results indicate a higher level of cognitive perceptual schizotypy than for those who do not have this experience. Possibly, these people have a much more intense imaginative life. This does not necessarily have any psychopathological implications for the individual; people who see auras might simply be sensitive to anomalous perceptual experiences.

Keywords: Aura; Fantasy prone; Absorption; Parasomatics; Imagery.
\end{abstract}

\section{Introducción}

La visión de aura ha estado tradicionalmente relacionada con el cuerpo etéreo sutil y sirve como una medida del estado de salud del cuerpo (Alfred, 2006). De acuerdo con las tradiciones occidentales, se cree que cada color del aura tiene un significado preciso que indiciaría un estado emocional específico (Leadbeater, 1902; Stanford, 1978; Swami Panchadasi, 1916). Algunos representantes de la Nueva Era identifican al aura, que presumiblemente ha sido demostrada mediante las fotografía Kirlian, como un campo

\footnotetext{
${ }^{1}$ Endereço para correspondência:

rapp@fibertel.com.ar
}

electromagnético que rodea a todos los seres vivos y a otros objetos inanimados (Krippner \& Rubin, 1974; Lindgren, 1995a, 1995b; Moss, 1979). Si bien el fenómeno del aura tiene una larga tradición en campos tales como la religión y el ocultismo, es controvertido determinar si los humanos pueden o no ver estos campos - tal vez como colores - a simple vista (Alvarado, 1987; Baltz \& Lindgren, 1997; Montandon, 1927, Perera Molina, 1981; Regush, 1977; Spence, 1920). Se pueden encontrar antecedentes de la observación del aura en una variedad de contextos, tanto en el campo de la hipnosis a veces el hipnotizado dice ver un un "fluído luminoso en los ojos y rodeando los dedos, la nariz, y las orejas del magnetizador" (Rochas, 1904; Kilner, 1965) hasta los relatos anecdóticos de diversos efectos luminosos en 
torno a místicos y santos (Alvarado, 1987; Zolla, 1994; Garrett, 1939; Karagulla, 1967). Butler (1978) clasificó el aura en dos tipos: etérico y espiritual. Bruce (2000) clasificó el aura en tres: etérico, central y espiritual.

Las tradiciones ocultistas postulan que la existencia de los cuerpos y energías sutiles desconocidas para la ciencia pero supuestamente percibidos por medios extrasensoriales (por ej., Leadbeater, 1902; Schwartz, 1980; para revisiones ver Bigu, 1976; Tart, 1972). Muchas observaciones anecdóticas sugieren que la "lectura del aura" puede ser relevante para el diagnósticos no convencionales, quizá sirviendo como un vehículo imaginario para la expresión (por ej., Karagulla, 1967) inaccesible a la conciencia. La experiencia de ver el aura también parece estar asociada al desarrollo de la sensibilidad psíquica después de las experiencias cercanas a la muerte (Greyson, 1983) así como también estar correlacionada con otros eventos paranormales, como percibir apariciones y tener experiencias fuera del cuerpo (Kohr, 1980; Palmer, 1979; Swann, 1975, pp. 21-22). La visión del aura también se ha relacionado con la investigación de presuntas radiaciones humanas en la historia de la investigación psíquica, como la búsqueda del un "fluido mesmérico" y la "fueza ódica" (Montandon, 1927; Sudre, 1975; Ungaro, 1992).

Sin embargo, las ilusiones perceptuales, los efectos de pos-imágenes, los efectos de contraste, o los fenómenos entópicos (la percepción de los spot o "emisiones" en la línea de visión, por la cual el individuo que la experimenta no tiene una explicación física) también se han presentado como una forma de explicar el efecto del aura (por ej., Dale 1961; Dale, Anderson \& Wyman, 1978; Fraser-Harris, 1932; Nash, 1986; Neher, 1980; Owen \& Morgan, 1974; Rawcliffe, 1952). Se han publicado muy pocos estudios sobre la experiencia de ver el aura asociada a variables psicológicas. En su mayoría tratan la fenomenología de la visión del aura. Por ejemplo, algunas encuestas mostraron la incidencia de esta experiencia en diferentes muestras. En estudios que han empleado muestras aleatorias, los porcentajes oscilan entre $0 \%$ a $7 \%$ (ver, Alvarado \& Zingrone, 1994; Haraldsson, Gudmundsdottir, Ragnarsson \& Jonsson, 1977; Palmer, 1979), pero en los grupos no aleatorios la incidencia del fenómeno fue algo más elevada, entre el $9 \%$ y $48 \%$.

Individuos que dicen ver el aura/energía en forma espontánea tienden a tener un nivel más elevado de actividad imaginativa/fantaseosa o propensidad a la fantasía. Esta hipótesis es coherente con la perspectiva de Wilson y Barber (1982), Blackmore (1978) y Siegel (1980) según la cual estas percepciones ocurren en el contexto de una fantasía alucinatoria que puede ser más fácilmente producida por individuos fantaseadores. Durante estos episodios, están completamente absortos en la experiencia (Tellegen, 1981). Parece haber, de hecho, una asociación entre los rasgos de suceptibilidad hipnótica y la apertura a la experiencia a causa de que los individuos involucrados en sus propios procesos mentales pueden estar más abiertos a ver aura o a tener otras experiencias psíquicas.

Cuando se compara a estos individuos con quienes no la experimentan, estos individuos tienen mayor capacidad de absorción psicológica (Glicksohn, 1990; Irwin, 1985; Myers, Austrin, Grisso \& Nickeson, 1983). Además, hay indicadores de que quienes experimentan alta capacidad de absorción, usualmente experimentan también diversas formas parasomáticas, alucinaciones táctiles, y sensaciones de energías sutiles (Irwin, 1985), y ocurren en el contexto de prácticas de meditación (Palmer \& Liberman, 1976). La propensidad a la fantasía parece ser un factor discriminante entre quienes ven el aura $y$ quienes no (Myers \& cols.., 1983; Wilson \& Barber, 1982). Una persona no necesita estar confundida o delirar para tener alucinaciones (Siegel, 1980; Slade, 1976; Slade \& Bentall, 1988).

No hay estudios en los cuales se haya investigado la relación entre ver el aura y los rasgos esquizotípicos, sin embargo, se puedo suponer que quienes puntúan significativamente alto en ideación esquizotípica, error perceptual, ideación mágica, y sinestesia en comparación con un grupo control, también indiquen tener experiencias de ver el aura. En tal sentido, la esquizotípia debería ser concebida como una dimensión de la personalidad distribuida continuamente en la población normal, cuya definición es que las personas en los extremos de la curva de distribución tienen una elevado grado de suceptibilidad a episodios psicóticos. La dimensión de personalidad esquizotípica puede estar correlacionada con una amplia variedad de otros fenomenos, además de las psicosis. En particular, hay buenos fundamentos para relacionar la esquizotipia con la creatividad (Claridge, Prior \& Watkins, 1989).

Por estas razones, creo que la visión del aura es parte de la experiencia humana y como tal merece ser estudiada en función de relacionarla 
con sus posibles componentes psicológicos y paranormales. Mi perspectiva es coherente con el planteo de Palmer (1979) y Alvarado y Zingrone (1997, 1999) acerca de la importancia de distinguir modelos convencionales de explicación de lo paranormal en parapsicología. Asi también, la necesidad de considerar los aspectos experimentales de psi como parte de la investigación parapsicológica sin necesariamente focalizar los modelos explicativos paranormales (Schouten, 1986; White, 1990). Parafraseando a Irwin (2004, p. 10),

"la experiencia bumana incluye un amplio rango de diferentes dimensiones de los cuales hay muchos aspectos que deben ser estudiados además de su ostensible paranormalidad."

Poco se conoce acerca de los factores psicológicos y los procesos que subyacen a la visión del aura, pero hay indicadores, en la literatura psicológica, parapsicológica y psiquiátrica, de que hay variables cognitivas (mentales) que merecen ser estudiadas.

\section{Hipótesis}

Este informe estudiará un número de estilos cognitivos (variables) tales como la absorción psicológica, las tendencias disociativas, la propensidad a la fantasía, la intensidad de la imaginería (particularmente asociadas a la ver el aura, por ej., las modalidades visual y táctil), la propensidad a alucinar (visual y táctil), y el estilo de personalidad propensa a la esquizotipia. Se ponen a prueba cuatro hipótesis específicas: que los estudiantes que ven el aura puntuarán más alto en (1) absorción, disociación, y propensidad a la fantasía, (2) intensidad de la imaginería, (3) propensidad a alucinar, (4) y esquizotipia cognitivo-perceptual en comparación con quienes no tienen la experiencia.

\section{Método}

\section{Participantes}

La muestra incluyó 678 estudiantes de psicología de ambos sexos, 449 (76,5\%) mujeres y $139(23,5 \%)$ varones, cuya rango etario es de $17 \mathrm{y}$ 57 años (Media $=25,54 ; \mathrm{SD}=7,22$ ) residentes en la Ciudad de Buenos Aires y alrededores. Ningun estudiante recibió compensación económica.

\section{Instrumentos}

Los estudiantes completaron seis escalas, cinco de éstas de experiencias perceptuales: 1) la
Escala de Imaginería (QMI, Sheehan, 1967; Richardson, 1990; alfa de Cronbach=0,77) que mide la intensidad de la imaginería en 35 items de una escala Likert 1-7, siendo 1 alta imaginería y 7 baja imaginería, en siete modalidades sensoriales (sólo dos se usaron: visual, por ej. "El sol poniéndose en el horizonte", y cinética, por ej. "Alcanzar un estante alto"); 2) el Cuestionario de Alucinaciones (CEA, Parra, Adróver y González, 2006; alfa de Cronbach $=0,93$ ) que mide la propensidad a alucinar en seis modalidades sensoriales de 38 items con una escala Likert 0-5, siendo 1 rara vez a 5 frecuentemente (sólo dos se usaron: visual, por ej. "He visto sombras, o figuras humanas o no-humanas cerca de mi cama, yo las he visto claramente y veo lo que hacen" y táctiles, por ej. "He tenido la experiencia de sentir una palmada en mi hombro, o cualquier otra sensación vívida de contacto físico de otra persona detrás mío, pero cuando me doy vuelta no veo a nadie"); 3) la Escala de Experiencias Disociativas (Bernstein y Putman, 1993; alfa de Cronbach $=0,91)$ una escala que mide una variedad de tendencias disociativas en tres factores: amnesia, despersonalización y desrealización en 28 items en una escala Likert 0-10 (por ej. "Algunas personas tienen la experiencia de conducir o estar viajando en un coche, colectivo, o subte y de repente se dan cuenta que no recuerdan lo que pasó durante todo o parte del viaje"); 4) el Cuestionario de Experiencias Creativas (CEQ, alfa de Cronbach=0,89, Merckelbach, Horselenberg y Muris, 2001) que mide la propensidad a la fantasía, especialmente la tendencia a fantasear en la niñez, en 25 ítems de respuesta verdadero/falso (por ej., "Cuando veo escenas de violencia por televisión, me siento tan involucrado en éstas que me provoca mucha inquietud"); 5) la Escala de Absorción de Tellegen (TAS, alfa de Cronbach $=0,91)$ (Tellegen \& Atkinson, 1974) que mide la frecuencia con que una persona se involucra en actividades que exigen atención y concentración en 34 ítems de respuesta verdadero/falso (por ej. "Cuando escucho música de órgano u otra música imponente a veces siento como si me estuvieran levantando en el aire").

Una medida tri-factorial de rasgos de personalidad utilizada fue el Cuestionario de Personalidad Esquizotípica (SPQ, alfa de Cronbach=0,91) (Raine, 1991; Raine, 1992, Raine \& Baker, 1992; Raine \& Benishay, 1995) que mide tres factores de esquizotipia en 74 ítems de respuesta dicotómica si/no (por ej. factor Cognitivo perceptual "¿Alguna vez ha visto cosas 
que para los demás son invisibles?” o “¿Son sus pensamientos a veces tan fuertes que usted casi podría escucharlos?", Interpersonal "Tengo poco interés en conocer a otras personas" o "Soy muy pobre al expresar mis verdaderos sentimientos por el modo en el que hablo y miro", y Desorganizado "Otras personas me ven como desatento o excentrico" o "A veces utilizo palabras de un modo inusual"). En general, un valor alfa de Cronbach de 0,60 es una medida aceptable de confiabilidad (Grady \& Wallston, 1988; Kim \& Mueller, 1978). Las medidas de alfa corresponden a la versión en español de cada escala.

Respecto a la experiencia de ver el aura, se formuló la pregunta de la siguiente manera: "¿Ha tenido alguna experiencia usted es capaz de ver campos de energia o luces alrededor del cuerpo de una persona? (si duda, por favor, responda no)". Si la respuesta es afirmativa, la pregunta también mapea tres dimensiones de la experiencia: frecuencia de la experiencia, explicación subjetiva (racional, desconocida y paranormal) de la experiencia, y positivo o negativo impacto (emocional) en una escala Likert, siendo 1 positivo y 7 negativo.

\section{Procedimiento}

Aplicamos una técnica de muestreo noprobabilística para obtener el mayor número de casos para analizar. El set de tests autoadministrables fue entregado en un sobre A4 a cada estudiante, en forma contrabalanceada, durante una clase de la cursada teórica. El tiempo promedio para completar los cuestionarios fue de
50 minutos. Los estudiantes recibieron una vaga información del objeto de estudio y se los invitó a participar voluntaria y anónimamente completando los tests, en una única sesión, en días y horarios previamente pactados con los docentes. El orden de administración de ambas pruebas fue contrabalanceado y los cuestionarios de alucinaciones se presentaron bajo el pseudo-título de Cuestionario de Experiencias Psicológicas, con lo cual se evitó sesgar las respuestas. Los análisis fueron procesados mediante el SPSS 11.5 (en español) y los análisis estadísticos fueron evaluados a dos colas.

\section{Resultados}

En base a las respuestas obtenidas para a pregunta de ver el aura se conformaron dos grupos:

Grupo aura: Para agrupar a quienes tenían la experiencia de ver el aura o "experientes" $[\mathrm{N}=83$ $(12,24 \%) \quad 28 \%$ varones y $68 \%$ mujeres, Edad Media $=24,12 ; \quad \mathrm{SD}=7,31]$ se convirtieron las respuestas 1 (rara vez), 2 (ocasionalmente), 3 (a menudo) y 4 (muy frecuentemente) a un valor (1).

Grupo "control" (no aura): Para agrupar a quienes no tuvieron EFC los ("no experientes") [ $\mathrm{N}=503$ $(87.76 \%), 22 \%$ varones y $78 \%$ mujeres, Edad Media $=24.92 ; \mathrm{SD}=7.26]$ se convirtió la respuesta "Nunca" a un valor (0).

Tabla 1 - Experiencia de ver el aura: frecuencia, explicación y impacto emocional

\begin{tabular}{llc}
\hline & & $\begin{array}{c}\text { Total } \\
(\mathrm{N}=83)\end{array}$ \\
\hline \multirow{2}{*}{ Frecuencia } & Una vez & $23(27,7)$ \\
& A veces & $48(57,8)$ \\
& Frecuentemente & $12(14,5)$ \\
\hline Impacto & Media $(1-7)^{(1)}$ & $2,44-1,47$ \\
Emocional & & \\
\hline \multirow{3}{*}{ Explicación } & Racional/Explicable & $28(35)$ \\
& Desconocida & $32(40)$ \\
& Paranormal/ & $23(25)$ \\
\hline
\end{tabular}

(1) 1 negativo a 7 positivo en el impacto emocional. 
Tabla 2 - Comparación de las medidas psicológicas de estudiantes que tienen la experiencia de ver el aura con aquellos que no la tienen(a)

\begin{tabular}{|c|c|c|c|c|c|}
\hline \multirow[b]{2}{*}{ Variables } & \multirow[b]{2}{*}{ Grupos } & \multicolumn{4}{|c|}{$\mathrm{Ude}$} \\
\hline & & Media & SD & Mann-Whitney & $\mathrm{z}$ \\
\hline \multirow[t]{2}{*}{ TAS } & Sin experiencia & 24,00 & 13,21 & 11640,0 & $2,67 * *$ \\
\hline & Con experiencia & 28,58 & 14,43 & & \\
\hline \multirow[t]{2}{*}{ DES } & Sin experiencia & 23,21 & 11,68 & 11531,0 & $2,77 *$ \\
\hline & Con experiencia & 26,68 & 12,3 & & \\
\hline \multirow[t]{2}{*}{ CEQ } & Sin experiencia & 31,89 & 15,01 & 9923,0 & $4,24 * * *$ \\
\hline & Con experiencia & 41,20 & 17,85 & & \\
\hline \multirow[t]{2}{*}{ QMI-KI } & Sin experiencia & 12,38 & 6,64 & 13699,5 & n.s. \\
\hline & Con experiencia & 11,53 & 6,05 & & \\
\hline \multirow[t]{2}{*}{ QMI-VI } & Sin experiencia & 13,56 & 7,16 & 14173,0 & n.s. \\
\hline & Con experiencia & 13,34 & 7,07 & & \\
\hline \multirow[t]{2}{*}{ CEA-VH(b) } & $\frac{1}{\text { Sin experiencia }}$ & 1,49 & 2,64 & 11843,5 & $2,75^{*}$ \\
\hline & Con experiencia & 3,88 & 4,35 & & \\
\hline \multirow[t]{2}{*}{ CEA-TH(b) } & Sin experiencia & 1,55 & 2,15 & 10722,5 & $3,79 * * *$ \\
\hline & Con experiencia & 3,57 & 3,99 & & \\
\hline \multirow[t]{2}{*}{ SPQ-CP } & Sin experiencia & 12,38 & 6,64 & 14173,0 & $4,50 * * *$ \\
\hline & Con experiencia & 11,53 & 6,05 & & \\
\hline \multicolumn{6}{|c|}{ 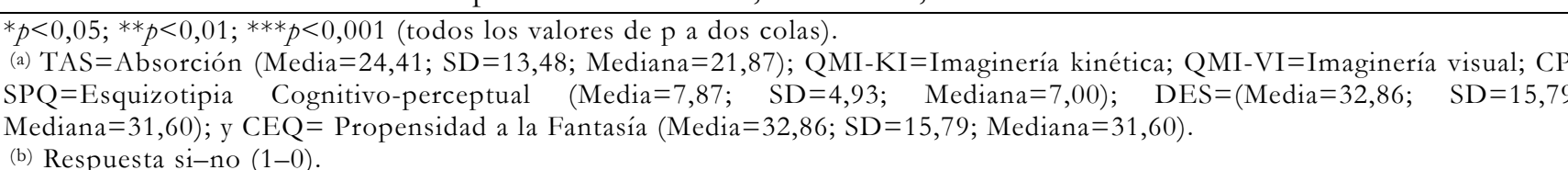 } \\
\hline
\end{tabular}

\section{Resultado de las hipótesis}

Absorción, Disociación, y Propensidad a la Fantasía: La Hipótesis 1 era que los estudiantes con experiencias puntuarían más alto en Absorción (medido con el TAS) que quienes no la tuvieron, Propensidad a la fantasía (medido con el CEQ), y Disociación (medido con el DES), la cual se confirmó: quienes tienen la experiencia puntuaron significativamente más alto (TAS $\mathrm{z}=2.77, \mathrm{p}<0,01$, a dos colas; DES $\mathrm{z}=2,67, \mathrm{p}<0,01$, a dos colas; CEQ $z=4,24, p<0,001$, a dos colas) que quienes no la tuvieron (ver Tabla 2).

Intensidad de la Imaginería. La Hipótesis 2 era que los estudiantes con experiencias puntuarían más alto en Imaginería visual e Imaginería kinética (medidos con el BIS) que quienes no la tuvieron, pero esta hipótesis no se confirmó para ninguna modalidad sensorial (ver Tabla 2).

Propensidad a alucinar. La Hipótesis 3 era que los estudiantes con experiencias puntuarían más alto en propensidad a alucinar (medidos con el CEA) que quienes no tuvieron la experiencia, la cual se confirmó: quienes tienen la experiencia puntuaron significativamente más alto (Visual $\mathrm{z}=2,75, \quad \mathrm{p}<0,05$, a dos colas, y Táctil $\mathrm{z}=3,79$, $\mathrm{p}<0,001$, a dos colas) que quienes no la tuvieron (ver Tabla 2).

Personalidad esquizotípica. La Hipótesis 4 era que los estudiantes con experiencias puntuarían más alto en el factor de esquizotipia cognitivo-perceptual (medidos con el SPQ) que quienes no la tuvieron, la cual se confirmó $(z=4,50, p<0,001$, a dos colas) (ver Tabla 2).

Para explorar la diferencia de géneros, se dividió en masculino/femenino y experientes/noexperientes, y se examinó el número de individuos que obtuvo puntuaciones sobre o por encima de la media, utilizando el test de probabilidad exacta de Fisher. El análisis de la frecuencia de las medidas psicológicas para los hombres en comparación con las mujeres no fue significativo en conjunto, así como también los experientes en comparación con los no-experientes examinados por separado. La diferencia entre ambos grupos no fue significativa. En otras palabras, no se encontró evidencia en cuanto a la diferencia por género en el conjunto de datos.

\section{Análisis de regresión logística}

¿Cuál de las diez variables discriminan mejor entre el grupo con experiencia y quienes no tuvieron la experiencia? Se llevó a cabo un análisis 
de regresión logística binaria para buscar el principal predictor. Parcialmente debido a un problema de colinealidad, después de verificar los requerimientos de la técnica, se llevó a cabo un análisis por el método de pasos hacia delante de Wald. Para reducir la colinealidad, se excluyeron del análisis de regresión algunas variables.

De una muestra de 563 participantes que reunían las condiciones, los resultados del mejor modelo (Paso 2) mostró que Alucinación visual era el mejor predictor para la experiencia de ver el aura (si/no) [Beta $=0,18$, Wald $=11,24 ; \mathrm{gl}=1$; $\mathrm{p}<0,001 ; \operatorname{Exp}(\mathrm{B})=1,20 ; \mathrm{R}^{2}$ de Nagelkerke's $=0,063$ ], $\mathrm{y}$ en segundo orden Esquizotipia cognitivoperceptual como predictora $[$ Beta $=0,05$, Wald=3,98; $\mathrm{gl}=1 ; \mathrm{p}<0,05 ; \operatorname{Exp}(\mathrm{B})=1,05 ; \mathrm{R}^{2} \mathrm{de}$ Nagelkerke $=0,075]$, pero ambas en un grado débil. Esto sugiere que la Esquizotipia cognitivoperceptual puede estar por debajo de la diferencia entre ambos grupos. De los dos predictores, solamente Alucinación visual tuvo una Beta levemente significativa $(0,18)$; mientras que el resto de las variables no resultaron predictoras. Sin embargo, la ver el aura parece ser una variable continua en el sentido de que la experiencia puede ocurrir más de una vez. Un análisis de la frecuencia de impacto (positivo) emocional (Media $=2,44 ; \mathrm{SD}=1,47$, siendo 1 positiva a 7 negativa) no resultó significativa.

\section{Discusión}

Este estudio examinó las diferencias entre aquellos que dicen ver el aura en comparación con aquellos que no la tienen en base a diferentes medidas cognitivas y de personalidad. Los resultados mostraron mayor nivel de esquizotipia cognitivo perceptual, absorción psicológica, tendencias disociativas, propensión a la fantasía, y alucinación visual y táctil, en comparación con quienes no tienen la experiencia de ver el aura. Posiblemente, quienes ven el aura son personas que tienen mucha más intensa vida imaginativa (Kohr, 1980; Myers, Austrin, Grisso \& Nickeson, 1983; Wilson \& Barber, 1983; Palmer, 1979). Tal descubrimiento sugiere que la visión del aura está relacionada con el proceso cognitivo-perceptual anómalo, la propensión a la fantasía y la alucinación visual.

Para Healy (1984), la percepción del aura y otros fenómenos como la sensibilidad de quien la experimenta es debido a los límites permeables del yo. Esta sensibilidad podría estar relacionada con la absorción o la disociación. Marks y McKellar
(1982) sugieren que la percepción del aura está asociada a alguna forma de imaginería eidética, la cual esta tiene un fundamento experimental utilizando tests basados en dibujos y diagramas, como los empleados por Matsuoka, Onizawa, Hatakeyama y Yamaguchi (1987). Se ha estudiado el efecto de persistencia de imagenes - mediante flashes de luz a los ojos de los pariticipantes - en relación a la suceptibilidad hipnótica y las habilidades visoespaciales (Atkinson \& Crawford, 1992). El resultado de este estudio también sugiere que el modelo de propensidad a la fantasía, que se cree subyace los prcesos alucinatorios como la absorción y la propensidad a la fantasía, estarían asociados con la experiencia de ver el aura.

Finalmente, también podemos concluir que una constelación de factores interrelacionados constituyen el constructo de la "personalidad propensa a la fantasía" (Wilson \& Barber, 1983), la cual predispone psicológicamente a la experiencia de ver el aura. Este tipo de experiencia también tiene implicaciones clínicas. Muchos terapeutas todavía consideran a estos pacientes (u otras experiencias asociadas a lo paranormal) como una enfermedad mental o un auto-engaño. Por esta razón, las personas propensas a fantasear, temen al ridículo y no le cuentan a nadie acerca de sus experiencias (Gómez Montanelli \& Parra, 2003, 2005; Tart, 1983a, 1983b, 1984). Pero esta experiencia no necesariamente posee un significado psicopatológico para el individuo, por lo cual no se debe concluir que la experiencia de ver el aura es patologica per se, no obstante un modelo disociacional sea necessario para dar cuenta de la habilidad cognitiva que presumiblemente subyace al estados de conciencia que pueden ser conducente a una variedad de experiencias perceptuales anómalas. Quienes ven el aura simplemente podrían ser sensibles a las experiencias perceptuales anómalas, pero a su vez, la disociación y absorción parecen estar presentes en la predisposición personal para tener la experiencia.

Un modelo teórico posible que surge de los resultados es el de la "esquizotipia feliz" (McCreery \& Claridge, 1995). El término alucinación aun tiene connotación peyorativa debido a que está asociado casi exclusivamente a la enfermedad mental o a estados anormales. Es interesante que algunos individuos pueden indicar efectos benéficos de la experiencia de ver el aura, por ejemplo, como que sirve como un medio visual del estado de la salud física y anímica, sanar a otros, o para mejorar la capacidad de ver el aura 
(Brennan, 1988). Si este es el caso, los individuos que tienen la experiencia de ver el aura deberían tambien experimentar desvios de otros mapas cognitivos, como las que presumiblemente subyacen en las experiencias de despersonalización. Esta idea surge a partir de los estudios que han encontrado una relación positiva entre las anomalías perceptuales (como la sinestecia) y una variedad de experiencias alucinatorias y de distorción perceptual.

\section{Referencias}

Alfred, J. (2006). Our invisible bodies: Scientific evidence for subtle bodies. Fairford, UK: Trafford.

Alvarado, C. S. (1994). Individual differences in aura vision: Relationship to visual imagery and imaginative-fantasy experiences. European Journal of Parapsychology, 10, 1-30.

Alvarado, C. S. \& Zingrone, N. (1987). Observations of luminous phenomena around the human body: A review. Journal of the Society for Psychical Research, 54, 38-60.

Atkinson, R. P. \& Crawford, H. J. (1992). Individual differences in afterimage persistence: Relationships to hypnotic susceptibility and visuospatial skills. American Journal of Psychology, 105, 527-539.

Baltz, J. \& Lindgren, C. E. (1997). Aura awareness: What your aura says about you. Nevada City, CA: Blue Dolphin.

Barrett, T. R. (1993). Verbal hallucinations in normals. Part 2: Self-reported imagery vividness Personality and Individual Differences, 15, 61-67.

Barrett, T. R. \& Etheridge, J. B. (1992). Verbal hallucinations in normals. Part 1: People who hear "voices". Applied Cognitive Psychology, 6, 379-387.

Bernstein, E. \& Putnam, F. (1986). Development, rehability, and validity of a dissociation scale. Journal of Nervous and Mental Disease, 174, 727735.

Bigu, J. (1976). On the biophysical basis of the human aura. Journal of Research in Psi Phenomena, 1, 8-43.

Blackmore, S. (1978). Parapsychology and out-of-thebody experiences. London: Transpersonal Books / Society for Psychical Research.
Brennan, B. A. (1988). Hands of light: A guide to bealing through the human energy field. Bantam Books.

Bruce, R. (2000). Auric mechanics and theory. Chicago, Ill: Blue Dolphin.

Butler, W. E. (1978). How to read the aura. Nova Iorque: Destiny Books.

Claridge, G. S., Pryor, R. \& Watkins, G. (1989) Sounds from the Bell Jar: Ten psychotic authors. Macmillan.

Dale, A., Anderson, D. \& Wyman, L. (1978). Perceptual aura: Not spirit but afterimage and border contrast effects. Perceptual and Motor Skills, 47, 653-654.

Fraser-Harris, D. F. (1932) A psycho-physiological explanation of the so-called human "aura". British Journal of Medical Psychology, 12, 174-184.

Garrett, E. (1939). My Life as a Search for the Meaning of Mediumship. Nova Iorque: Oquaga.

Glicksohn, J. (1990). Belief in the paranormal and subjective paranormal experience. Personality and Individual Differences, 11, 675-683.

Gómez Montanelli, D. \& Parra, A. (2003). Un abordaje modelo para el procesamiento de las reacciones emocionales ante experiencias paranormales. Revista Argentina de Psicología Paranormal, 14, 9-27.

Gómez Montanelli, D. \& Parra, A. (2005). ¿Las Experiencias Paranormales son psicológicamente perturbadoras? Una encuesta comparando estudiantes universitarios y aficionados a temas paranormales. Revista Interamericana de Psicología, 39, 285-294.

Greyson, B. (1983). Increase in psychic phenomena following near-death experiences. Theta, 11, 26-29.

Haraldsson, E., Gudmundsdottir, A., Ragnarsson, J. L. \& Jonsson, S. (1977) National survey of psychical experiences and attitudes towards the paranormal in Iceland [Abstract]. En J. D. Morris, W. G. Roll, \& R. L. Morris (Orgs.). Research in Parapsychology 1976 (pp. 182-186). Metuchen, NJ: Scarecrow.

Healy, J. (1984). The happy princess: Psychological profile of a psychic. Journal of the Society for Psychical Research, 52, 289-296. 
Irwin, H. J. (1985). Flight of mind: A psychological study of the out-of-body experience. Metuchen, NJ: Scarecrow.

Irwin, H. J. (1988). Out-of-body experiences and attitudes to life and death. Journal of the American Society for Psychical Research, 82, 237 251.

Irwin, H. J. (2004). An introduction to parapsychology ( $4^{\mathrm{a}}$ Ed.). Jefferson, NC: McFarland.

Krippner, S. \& Rubin, D. (1974). The Kirlian aura: photographing the galaxies of life. Anchor Press/Doubleday.

Karagulla, S. (1967). Breakthrough to creativity: Your higher sense perception. Los Angeles: DeVors.

Kilner, W. J. (1920). The human atmosphere. (2 ed.). London: Kegan Paul, Trench, Trubner.

Kohr, R. L. (1980) A survey of psi experiences among members of a special population. Journal of the American Society for Psychical Research, 74, 395-411.

Krippner, S. \& Rubin, D. (1973). Galaxies of life. The buman aura in acupuncture and Kirlian photography. Nova Iorque: Cordon and Breach.

Leadbeater, C. W. (1902). Man visible and invisible: Examples of different types of men as seen by means of trained clairvoyance. New York, NY. John Lane.

Lindgren, C. E. (1995a). A review of aura imaging photography by Johannes Fisslinger. Journal of Religion and Psychical Research, 18, 49-50.

Lindgren, C. E. (1995b). Capturing your aura on film. Fate, 48(1), 32-35.

Matsuoka, K., Onizawa, I., Hatakeyama, T. \& Yamaguchi, H. (1987). Incidence of young adult eidetikers, and two kinds of eidetic imagery. Tohoku Psychologica Folia, 46, 6274.

McCreery, C. \& Claridge, G. (1995). Out-of-the body experiences and personality. Journal of the Society for Psychical Research, 60, 129 -148.

McKelvie, S. J. (1979). Effects of instructions and format on reported visual imagery. Perceptual and Motor Skills, 49, 567-571.

Montandon, R. (1927). Les radiations bumaines. Paris: Félix Alcan.

Moss, T. (1979). The body electric: A personal journey into the mysteries of parapsychological research, bioenergy, and Kirlian photography, Los Angeles, CA: Tarcher.

Murphy, G. \& Dale, L. A. (1961). Challenge of psychical research: A primer of parapsychology. Nova Iorque: Harper \& Row.

Myers, S. A. (1983). The Wilson-Barber Inventory of Childhood Memories and Imaginings: Children's form and norms for 1337 children and adolescents. Journal of Mental Imagery, 7, 83-94.

Palmer, J. (1979). A community mail survey of psychic experiences. The Journal of the American Society for Psychical Research, 73(3), 221-251.

Myers, S. A., Austrin, H. R., Grisso, J. T. \& Nickeson, R. C. (1983). Personality characteristics as related to the out-of-body experience. Journal of Parapsychology, 47, 131144.

Nash, C. B. (1986). Parapsychology: The science of psiology. Springfield, Il.: Charles C. Thomas.

Neher, A. (1980). The psychology of transcendence. Englewood Cliffs, NJ: Prentice-Hall.

Owen, A. R. G. \& Morgan, G. A. V. (1974). The "rim" aura, an optical illusion: A genuine but non-psychic perception. New Horizons, 1,19-31.

Palmer, J. (1979). A community mail survey of psychic experiences. Journal of the American Society for Psychical Research, 73, 221-251.

Palmer, J. \& Lieberman, R. (1976). ESP and outof-body experiences: A further study. Research in Parapsychology 1975, 102-106.

Pekala, R., Kumar, V. K. \& Cummings, J. (1992). Types of high hypnotically susceptible individuals and reported attitudes and experiences of the paranormal and the anomalous. Journal of the American Society for Psychical Research, 86, 135-150.

Perera Molina, R. (1981) Del aura a la cámara Kirlian. In L. Fernández Briones (Ed.), La Nueva Parapsicología: Introducción a la Parapsicología Cientifica (pp. 272-290). Barcelona: Noguer.

Raine, A. (1991). The SPQ: A scale for the assessment of schizotypal personality based on DSM-III-R criteria. Schizophrenia Bulletin, 17, 556-564. 
Raine, A. (1992). Sex differences in schizotypal personality in a non-clinical population. Journal of Abnormal Psychology, 101, 361-364.

Raine, A. \& Baker, L. (1992) The Schirotypal Personality Questionnaire: Genetics, Psychophysiology. Neuropsychology and Gender Differences. Western Psychological Association, Portland, Oregon.

Raine, A. \& Benishay, D. (1995). The SPQ-B: A brief screening instrument for schizotypal personality disorder. Journal of Personality Disorders, 9, 346-355.

Rawcliffe, D. H. (1952). The psychology of the occult. London: Derricke Ridgeway.

Regush, N. (1977). Exploración del aura bumana. México, D.F.: Diana.

Rochas, A. de (1904). Les états profonds de bypnose, 5th ed. Paris: Chacornac.

Richardson, A. (1990). Mental imagery. New York, NY: Springer.

Schouten, S. A. (1986) A different approach for studying psi. En B. Shapin \& L. Coly (Orgs.), Current Trends in Psi Research (pp. 27-39). New York, NY: Parapsychology Foundation.

Schwartz, J. (1980). Human Energy Systems. Nova Iorque: E.P. Dutton.

Sheehan, P. W. (1967). A shortened forms of Betts' Questionnaire upon Mental Imagery. Journal of Clinical Psychology, 23, 386-389.

Siegel, R. K. (1980). The psychology of life after death. American Psychologist, 35, 911-931.

Slade, P. D. \& Bentall, R. P. (1988). Sensory Deception. London: Croom Helm.

Slade, P. D. (1976). An investigation of psychological factors involved in the predisposition to auditory hallucinations. Psychological Medicine 6, 123 -132.

Spence, L. (1920) An encyclopaedia of occultism. London: George Routledge.

Stanford, R. (1978). El conocimiento del aura. Buenos Aires: Troquel

Sudre, R. (1975). Tratado de Parapsicología. Buenos Aires: Siglo XX.

Swami Panchadasi (1916). The human aura. Astral colors and thought forms. Chicago, Ill: Advanced Thought Publishing.
Swann, I. (1975). To Kiss Earth Good-bye. New York. Hawthorne Books.

Tart, C. T. (1972). Concerning the scientific study of the human aura. Journal of the Society for Psychical Research, 46, 1-21.

Tart, C. T. (1983a). The controversy about psi: Two psychological theories. Journal of Parapsychology, 46, 313-320.

Tart, C. T. (1983b). Who's afraid of psychic powers? Me? The Open Mind, 1(3), 1-5.

Tart, C. T. (1984). Acknowledging and dealing with the fear of psi. Journal of the American Society for Psychical Research, 78, 133-143.

Tellegen, A. (1981). Practicing the two disciplines for relaxation and enlightenment: Comment on "Role of the feedback signal in electromyograph biofeedback: The relevante of attention by Qualls and Sheehan". Journal of Experimental Psychology, 110(2), 217-226.

Tellegen, A. \& Atkinson, G. (1974). Openness to absorbing and self altering experiences ("absorption"), a trait related to hypnotic susceptibility. Journal of Abnormal Psychology, 83, 268-277.

Ungaro, J. (1992). Las radiaciones humanas: Historia de la radiónica. Buenos Aires: Club de Estudio.

White, R. A. (1990) An experience-centered approach to parapsychology. Exceptional Human Experience: Studies of the Psychic/Spontaneous/Intangible, 8, 7-36.

Wilson, S. C. \& Barber, T. X. (1978) The Creative Imagination Scale as a measure of hypnotic responsiveness: Applications to experimental and clinical hypnosis. American Journal of Clinical Hypnosis, 20, 235-249.

Wilson, S. C., \& Barber T. X. (1982). The fantasyprone personality: Implications for understanding imagery, hypnosis, and parapsychological phenomena. In A.A. Sheikh (Ed.) Imagery: Current theory, research, and application. New York: John Wiley.

Zolla, E. (1994). Auras: Culturas, lugares y ritos. Barcelona. Paidós.

Recebido em novembro de 2007

Reformulado em maio de 2008

Aprovado em setembro de 2008 
Sobre o autor:

Alejandro Parra é psicólogo e docente de Psicologia na Faculdade de Psicologia da Universidad Abierta Interamericana. Presidente do Instituto de Psicología Paranormal de Buenos Aires (www.alipsi.com.ar), e autor de três livros, o mais recente, Psicología de las Experiencias Paranormales: Introducción a la teoría, investigación y aplicaciones terapéuticas 